\title{
Uso de GH em Pacientes com Baixa Estatura Idiopática
}

revisão

\section{Carlos Alberto longui}

Laboratório de Medicina Molecular do Departamento de Ciências Fisiológicas da Faculdade de Ciências Médicas da Santa Casa de São Paulo e Unidade de Endocrinologia Pediátrica do Departamento Pediatria e Puericultura da Irmandade da Santa Casa de Misericórdia de São Paulo, SP,

Brasil.

Recebido em 15/4/2008 Aceito em 21/4/2008

\section{RESUMO}

Pacientes com baixa estatura idiopática podem ser tratados com GH. Os critérios clínicos e laboratoriais utilizados na decisão do uso do $\mathrm{GH}$, bem como no reconhecimento da responsividade dos indivíduos ao tratamento, são discutidos. Não apenas os resultados antropométricos, mas também os aspectos éticos e psicossociais devem ser considerados na avaliação dos custos/ benefícios envolvidos no tratamento com $\mathrm{GH}$ em pacientes com baixa estatura idiopática. (Arq Bras Endocrinol Metab 2008; 52/5:750-756)

Descritores: Baixa estatura idiopática; Tratamento da baixa estatura; Hormônio de crescimento; $\mathrm{GH}$; Baixa estatura familial; Retardo constitucional do crescimento e da puberdade

\section{ABSTRACT}

\section{GH Treatment in Patients with Idiopathic Short Stature.}

Growth hormone has been used in the treatment of patients with idiopathic short stature. Clinical and laboratorial criteria are discussed, taking into consideration the indication of GH and the evaluation of its efficacy and individual responsiveness. Anthropometric, psychosocial, ethical, and also cost/ benefit aspects must be considered before $\mathrm{GH}$ prescription in idiopathic short stature patients. (Arq Bras Endocrinol Metab 2008; 52/5:750-756)

Keywords: Idiopathic short stature; Short stature therapy; Growth hormone; Familial short stature; Constitutional delay of growth and puberty

$\mathrm{O}$ TRATAMENTO DA BAIXA ESTATURA (BE) com hormônio de crescimento humano $(\mathrm{GH})$ tem sido utilizado há várias décadas. Inicialmente em pacientes com deficiência de GH e, logo a seguir, em doenças genéticas, como a síndrome de Turner, e em doenças crônicas, como a insuficiência renal. Nesta mesma época, indivíduos com estatura abaixo do normal para a idade e o sexo, ou abaixo do esperado para o padrão familial (estatura-alvo familial ou target height, TH) também passaram a ser tratados com GH (1). Hoje, a experiência clínica obtida com o tratamento de centenas de milhares de pacientes com BE permite avaliar os riscos e os benefícios envolvidos no uso de $\mathrm{GH}$, bem como reconhecer os principais fatores determinantes da resposta terapêutica (2).

Um número significante de pacientes tratados com GH corresponde a crianças e adolescentes com baixa estatura idiopática (BEI). Trata-se de grupo heterogêneo, que inclui três subclasses de diagnóstico: a baixa estatura familial (BEF), o retardo constitucional do crescimento e puberdade (RCCP) e a BEI propriamente dita (3). Na prática, uma parcela substancial de casos apresenta elementos comuns a mais de uma subclasse diagnóstica. Isso se deve ao 
fato de existir um espectro de variação na estatura dos pais, na maturação óssea e no momento de início e rapidez evolutiva do evento puberal.

\section{BAIXA ESTATURA FAMILIAL (BEF)}

Pacientes com BEF encontram-se caracteristicamente no percentil médio dos pais, os quais também são baixos em relação à população geral. Um fator complicante nesta situação é a freqüente diferença de estatura entre os pais, o que determina que os filhos possam estar mais próximos do percentil de um dos pais e não necessariamente na média deles. Além disso, na definição da estatura-alvo, deve-se considerar, em nosso meio, que uma aceleração secular do crescimento populacional ainda esteja ocorrendo, o que determina o incremento de cerca de $4,5 \mathrm{~cm}$ na estatura final atual em relação à geração anterior. Este aspecto pode ser relevante na definição de perda de estatura por ocasião da previsão de crescimento de determinado paciente. Nos pacientes com BEF, a velocidade de crescimento (VC) é normal ou no limite inferior do normal, a idade óssea (IO) compatível com a idade cronológica (IC) e a previsão de estatura final (pEf) compatível com o TH, ou seja, abaixo do normal para a população geral.

\section{RETARDO CONSTITUCIONAL DO CRESCIMENTO E PUBERDADE (RCCP)}

Pacientes com RCCP apresentam-se em percentis de estatura inferiores ou abaixo da população geral e também abaixo do percentil dos pais. A VC costuma ser normal/ baixa (entre os percentis 10 e $25 \mathrm{da} \mathrm{VC}$, ou seja, ao redor de $4 \mathrm{~cm} /$ ano). Caracteristicamente, a IO é atrasada em relação à IC, o que confere uma pEf compatível com o padrão familial. O início puberal é atrasado em relação à media populacional, sendo freqüente a história familial de atraso puberal. Esta situação apresenta várias peculiaridades que a tornam de difícil manejo clínico. O diagnóstico de RCCP é sempre de exclusão e, portanto, fundamentado no fato de que doenças genéticas ou crônicas sistêmicas tenham sido afastadas. Além disso, sua confirmação diagnóstica só será possível de maneira retrospectiva, quando, ao término do acompanhamento, a estatura final for compatível com o padrão familial. Várias anormalidades genéticas ou doenças crônicas podem ser oligossintomáticas e de manifestação lentamente progressiva, permanecendo como RCCP até que a suspeita clínica ou a triagem rotineira possa identificar o diagnóstico etiológico subjacente. Isso ocorre, por exemplo, em casos de insuficiência renal crônica, acidose tubular renal (especialmente em crianças menores), doença celíaca ou doença inflamatória intestinal, em pacientes com alterações cromossômicas e fenótipo menos severo e nos casos de deficiências hormonais menos intensas, como nos de evolução insidiosa de hipotireoidismo ou deficiências parciais de GH. Ainda nos pacientes que se apresentam inicialmente com RCCP, outros fatores podem comprometer a estatura final, pois alguns pacientes apresentam de maneira inesperada para a IO uma puberdade em IC normal. Além disso, boa parte dos pais de pacientes com RCCP também apresentam percentis de estatura normal, porém inferior à média da população geral, fazendo que a ocorrência de perda relativamente pequena da estatura, em relação ao $\mathrm{TH}$, possa determinar estatura final do paciente significativamente baixa em relação à população geral.

\section{BAIXA ESTATURA IDIOPÁTICA PROPRIAMENTE DITA}

Um número considerável de crianças encontram-se abaixo do percentil dos pais, apresentando VC normal/ baixa, IO compatível com a IC ou pouco atrasada, com pEf abaixo do TH e puberdade em época normal para a população geral. É provável que estes pacientes atingirão estatura final inferior à dos pais e, freqüentemente, baixa estatura em relação à população geral. Nestes casos, a investigação etiológica não permite identificar a causa da perda estatural e o quadro permanece verdadeiramente idiopático ao longo do seguimento clínico. Às vezes, esta situação é de difícil diagnóstico diferencial com o retardo de crescimento intra-uterino, pois ao redor de um terço dos pacientes com BEI nascem com comprimento reduzido. A análise da curva de crescimento dos primeiros 2 a 3 anos de vida pode auxiliar no diagnóstico diferencial. Enquanto nas crianças nascidas pequenas para a idade gestacional existe apenas recuperação parcial da estatura ao final deste período, com manutenção do canal de crescimento abaixo do normal nos anos que se seguem, em pacientes com BEI que nascem pequenos existe recuperação completa do crescimento na fase inicial, seguida de período de desaceleração idiopática do crescimento precedendo a avaliação clínica atual.

De modo geral, a BEI representa cerca de $80 \%$ de todos os pacientes com $\mathrm{BE}$ que procuram atendimento 
médico. O percentil de estatura é inferior ao p2,5 (ou seja, abaixo de $-2,0 \mathrm{DP}$ ). Deve-se considerar que quando os pais estão acima do p50 (DP 0), filhos que se encontram um a dois desvios abaixo do padrão dos pais também devem ser avaliados e o tratamento considerado.

$\mathrm{O}$ diagnóstico de BEI deve ser de exclusão, considerando-se a história clínica cuidadosa e o exame físico detalhado que inclua dados antropométricos, como estatura, peso, IMC e relação entre os seguimentos superior e inferior, também evidenciada pela relação da estatura sentada em comparação à estatura do paciente (4). São ainda necessários exames laboratoriais de triagem, como hemograma, VHS, proteínas totais e frações, creatinina, cálcio, fósforo e fosfatase alcalina, gasometria venosa, enzimas hepáticas, anticorpos antitransglutaminase ou antiendomísio, cariótipo, T4, TSH, IGF-1 e idade óssea. Em casos específicos, o diagnóstico de BEI poderá requerer o diferencial com a insuficiência hipofisária, quando os testes de estímulo do GH e a ressonância nuclear magnética (RNM) hipotálamo-hipofisária poderão ser necessários. Vários estudos têm apontado quadros de secreção anormal de GH em pacientes com BEI, ou mesmo a presença de quadros de resistência parcial ao efeito do GH ou do IGF-1 $(5,6)$. Os testes diagnósticos para estas situações são pouco reprodutíveis, o que dificulta sua aplicabilidade no diagnóstico diferencial destes casos. Quando um dos pais é baixo, deve-se admitir a possibilidade da presença de doença hereditária de caráter dominante. É possível que, no futuro, a disponibilidade de estudos moleculares possa permitir o reconhecimento de alterações gênicas que isoladamente ou em conjunto determinem a perda estatural (7). Com isso, pacientes que hoje são classificados como portadores de BEI passarão a ter o diagnóstico etiológico conhecido.

A época de início puberal é um dos fatores determinantes da estatura final. Nos pacientes com BEI, assim como na população geral, existe um espectro de idade no qual ocorre o início puberal. Isso faz que, de um lado, pacientes com BEF e avanço puberal fiquem ainda menores que seus pais, e, de outro, pacientes com BEF e atraso puberal possam recuperar a estatura ou mesmo superar o TH. Da mesma forma, pacientes com RCCP e atraso característico do início puberal podem atingir seu $\mathrm{TH}$, enquanto aqueles que inesperadamente entram em puberdade antes da média populacional acabam por não atingir a pEf inicialmente calculada ou seu TH. Em pacientes com BEI, o melhor cenário ocorre em casos com adequado $\mathrm{TH}$, atra- so da IO e atraso puberal, enquanto o pior cenário é observado nos casos com estatura familial baixa, IO compatível com a IC e puberdade em idade inferior à média populacional ( 11 e 12 anos na menina; 12 e 13 anos no menino).

Esta subclassificação das crianças com BEI auxilia na decisão do uso de $\mathrm{GH}$, sendo ainda importante na identificação dos casos de maior ou menor potencial de resposta clínica ao tratamento com GH.

\section{TRATAMENTO DE PACIENTES COM BEI}

$\mathrm{Na}$ decisão terapêutica de pacientes com BEI diversos aspectos devem ser considerados, entre os quais a severidade da $\mathrm{BE}$, o prognóstico de estatura final, bem como os aspectos psicossociais que envolvem o paciente e sua família (4).

Enquanto os aspectos antropométricos são facilmente determinados, a pEf tem baixa acurácia e é freqüentemente influenciada por fatores que induzem a subestimar ou superestimar a estatura final (8).

A visão dos pais sobre a possibilidade da estatura final reduzida também é muito variável, influenciando o comportamento da própria criança e a decisão terapêutica. $\mathrm{O}$ impacto psicossocial exercido pela $\mathrm{BE}$ não está plenamente estabelecido, especialmente em nosso meio. Os estudos são discordantes quanto ao comportamento adaptativo de crianças com $\mathrm{BE}$ em resposta às pressões sociais, por exemplo, serem alvos de brincadeiras pejorativas ou receberem tratamento social infantilizado ou com baixa expectativa em virtude de sua estatura. Alguns índices de ajuste psicossocial inadequado são citados, como auto-imagem e auto-estima alterados, porém são muito variáveis entre os diferentes pacientes. Em boa parte, esses achados contraditórios evidenciam a falha das ferramentas diagnósticas utilizadas para esse tipo de avaliação.

Alguns aspectos éticos importantes devem ser considerados, entre os quais a severidade da BE que justifique a indicação do tratamento. Além disso, deve-se considerar o potencial benefício para a criança e não a pressão exercida pela angústia dos pais. Deixar claro à família e ao paciente o real impacto que o tratamento pode ter sobre a estatura final, com grande espectro de respostas, significando que parte dos pacientes poderá não apresentar benefício satisfatório ao final da terapia, especialmente se a expectativa de grande incremento estatural estiver presente. 
Embora a decisão seja sempre difícil pela presença dos diversos aspectos citados anteriormente, é necessário que tanto o médico quanto a família tenha ciência de que a precocidade com que o tratamento seja instituído talvez seja o principal fator determinante de sua eficiência. Portanto, aguardar um tempo longo para que exista certeza de um prognóstico estatural ruim pode determinar baixa eficiência ou mesmo a impossibilidade que o tratamento seja realizado.

\section{USO DE GH EM PACIENTES COM BEI}

Existe grande número de estudos observacionais e de intervenção com GH realizados em serviços que atendem crianças e adolescentes com BE. Porém, a maior parte deles apresenta divergência de aspectos críticos, que não permite a comparação entre os resultados. Os critérios de inclusão de pacientes são muito variáveis, com grande discrepância entre a severidade da $\mathrm{BE}$, a idade de início do $\mathrm{GH}$, as diferentes doses de $\mathrm{GH}$, os pacientes em estágios puberais distintos ou que desenvolveram a puberdade durante o tratamento, a ausência de grupos-controle adequados, entre outros aspectos. Dessa forma, os estudos mais antigos e não bem controlados para as diferentes variáveis apresentam desde ausência de resposta ao $\mathrm{GH}$ até respostas que superam 2 DP $(14 \mathrm{~cm})$ de ganho na estatura final.

Revisões mais recentes como as de Bryant e cols. (9) e a de Quigley (10), publicadas em 2007, apresentam estudos com maior nível de evidência clínica e permitem reconhecer com maior precisão o impacto do GH no tratamento da BEI. A VC no primeiro ano de tratamento representa bom índice de sensibilidade ao $\mathrm{GH}$ (11), e em pacientes em fase pré-puberal habitualmente sofre um incremento entre $50 \%$ e $100 \%$ da VC pré-tratamento. Tal resposta está diretamente relacionada à dose de GH utilizada e inversamente relacionada à idade de início do tratamento, ou seja, a melhor resposta é observada em pacientes mais novos, portanto, ainda distantes da puberdade. O incremento da VC observado em pacientes com BEI é similar em padrão e amplitude ao obtido em pacientes com síndrome de Turner ou os que nasceram pequenos para a idade gestacional.

A dose de GH empregada em pacientes com BEI é geralmente suprafisiológica $(12,13)$, variando entre 0,15 e $0,20 \mathrm{UI} / \mathrm{kg} /$ dia $(0,05$ a $0,066 \mathrm{mg} / \mathrm{kg} / \mathrm{dia})$. Nestas doses, a maior parte dos estudos não reporta avanço significante da IO ou antecipação puberal. Tais efeitos adversos já foram relatados quando doses maiores foram utilizadas, embora haja grande variabilidade individual no avanço da IO (14). Em estudos de longa duração com avaliação da estatura final, o ganho de estatura encontra-se significantemente relacionado à dose utilizada. Doses de $\mathrm{GH}$ semelhantes às utilizadas em deficientes de $\mathrm{GH}$, ou seja, ao redor de $0,10 \mathrm{UI} / \mathrm{kg} /$ dia $(0,033 \mathrm{mg} / \mathrm{kg} /$ dia $)$ determinam ganho discreto na estatura final ao redor de $3 \mathrm{~cm}$, mesmo quando esta dose é aumentada posteriormente na época puberal. Um ganho médio de $7 \mathrm{~cm}$ na estatura final foi observado em pacientes com BEI tratados com GH (15) na dose de $0,15 \mathrm{UI} / \mathrm{kg} /$ dia $(0,05 \mathrm{mg} / \mathrm{kg} /$ dia $)$. O início precoce do tratamento com $\mathrm{GH}$, bem como o uso de doses iniciais mais elevadas parecem ser críticas na obtenção de resposta terapêutica mais adequada.

Efeitos metabólicos durante o uso de GH também são observados em pacientes com BEI e incluem aumento da massa magra e redução da massa gordurosa com melhora do perfil lipídico.

$\mathrm{O}$ reconhecimento dos efeitos psicossociais determinados pelo tratamento com GH sofre as mesmas limitações metodológicas encontradas para a definição do impacto psicossocial da BE.

Diversos fatores são determinantes da resposta terapêutica ao uso de $\mathrm{GH}$ em pacientes com BEI, incluindo substancial variação na sensibilidade entre os indivíduos. Entre os principais fatores que determinam a amplitude de resposta ao $\mathrm{GH}$, durante o primeiro ano de tratamento, pode-se destacar a IC (16) ao início do GH (melhor quanto menor a IC), a dose do GH (melhor quanto maior a dose) e a severidade da BE em relação ao TH (melhor resposta quanto mais abaixo do $\mathrm{TH}$ ). A resposta terapêutica em longo prazo é influenciada pelos mesmos fatores, adicionados da estaturaalvo familial (melhor resposta quanto maior o TH). Os resultados laboratoriais são muito variáveis e não são utilizados como critérios de avaliação da resposta terapêutica, pois apresentam menor capacidade de predição de resposta em longo prazo, tendo, portanto, pouca aplicabilidade clínica. A tentativa de tratamento da BEI com GH em pacientes já em plena fase puberal apresenta baixa eficiência. Vários estudos realizados nesta fase propõem a associação de bloqueadores da puberdade (17), como os GnRH agonistas de ação prolongada e/ ou inibidores da aromatase $(18,19)$. O bloqueio puberal com os GnRHa, especialmente os de depósito, são eficazes no controle da progressão puberal, permitindo maior tempo de uso de GH. Sua eficácia em promover 
ganho estatural satisfatório é maior quando iniciado nas fases precoces do desenvolvimento puberal, adicionando ganho médio de 4 a $5 \mathrm{~cm}$ na estatura final. A tentativa de bloqueio puberal em fases mais avançadas ou com maior IO é menos efetiva e está associada ao risco inerente de supressão da função de uma gônada já em fase avançada de maturação e que poderá não se recuperar plenamente após a suspensão do tratamento. O uso de inibidores de aromatase em associação ao $\mathrm{GH}$ pode ser potencialmente útil em meninos, pois, ao reduzirem a velocidade de maturação óssea, retarda o fechamento da cartilagem de crescimento, o que permite maior tempo de tratamento com GH. Por outro lado, a experiência com os inibidores da aromatase é ainda inicial, não havendo estudos de longo prazo, especialmente sobre a segurança de uso em relação aos possíveis efeitos adversos sobre a espermatogênese da vida adulta.

Maior tempo de uso do GH está habitualmente relacionado a maior ganho na estatura final. Porém, o prolongamento da terapia com GH acima de determinado ponto passa a ser de pouco benefício e adiciona custos e riscos desnecessários, podendo ainda gerar crescimento desproporcional das extremidades e risco de processo compressivo de nervos periféricos distais. A VC inferior a $2 \mathrm{~cm} /$ ano tem sido um limitante para a continuidade do uso de $\mathrm{GH}$, habitualmente presente em meninas com IO superior a 14 anos ou meninos com IO superior a 16 anos.

\section{CRITÉRIOS DE CONTROLE DO TRATAMENTO}

A VC ajustada ao estágio de atividade puberal e à IO é importante ferramenta auxológica na avaliação da responsividade ao tratamento com $\mathrm{GH}$ em pacientes com BEI. Além disso, serve para alertar sobre a possibilidade de baixa adesão ao tratamento ou aparecimento de doenças intercorrentes. É sempre conveniente ressaltar que a causa da BEI pode apresentar-se mais tardiamente e de maneira insidiosa.

A IO é avaliada a cada 6 meses, com o objetivo de detectar avanço desproporcional que possa significar início puberal inadequadamente precoce. Além disso, a IO permite o cálculo de pEf subseqüentes, que embora tenham baixa precisão em determinar a estatura real a ser obtida no final do crescimento, são úteis no reconhecimento da tendência de ganho ou perda durante o tratamento. A radiografia de quadril (AP) pode ser útil na avaliação do ângulo de Southwick, que representa fator de risco quando estiver aumentado em relação ao normal (20).

Os controles hormonais semestrais incluem as quantificações da glicemia de jejum e da insulinemia, que representam exames de triagem na detecção de casos que evoluam com significante resistência insulínica durante o tratamento com GH. Valores de T4 e TSH também devem ser monitorados ao menos anualmente. A determinação semestral de IGF-1 e IGFBP-3 pode auxiliar na identificação da responsividade ao $\mathrm{GH}$, adesão ao tratamento e mesmo oferecer subsídio para maior segurança e eficiência na escolha da dose terapêutica a ser empregada. Ainda em fase experimental clínica está a proposta de ajuste da dose de GH objetivando-se a elevação da concentração de IGF-l a valores superiores à media normal e inferiores a $+2 \mathrm{DP}$, para idade, sexo e estágio puberal ou IO do paciente. Este alvo terapêutico evita o uso de dose baixa de GH que, ao gerar pouca IGF-1, induz resposta terapêutica insuficiente, ao mesmo tempo que evita o uso de dose elevada de $\mathrm{GH}$, capaz de gerar concentrações persistentemente altas de IGF-1 com potencial risco de efeitos metabólicos adversos e exagerada proliferação celular.

\section{EFEITOS ADVERSOS}

O tratamento com GH tem se mostrado seguro em estudos de curta e longa duração (15). Em pacientes com BEI, efeitos adversos mais sérios como hipertensão intracraniana benigna, pancreatite ou desenvolvimento de tumores são virtualmente ausentes. Deve-se, porém observar e valorizar a presença de cefaléia refratária ao uso de analgésicos comuns ou acompanhada de náuseas ou alterações visuais. Nestes casos, as avaliações oftalmológica e neurológica são essenciais. $\mathrm{O}$ escorregamento epifisário do fêmur proximal tem sido raramente descrito em pacientes com BEI ( $1 \mathrm{em}$ 10.000 tratados). Anormalidades do metabolismo de hidratos de carbono também são pouco freqüentes, mas devem ser investigadas, especialmente em pacientes obesos ou com sugestiva história familial de resistência insulínica. Não há evidências de que tais efeitos metabólicos representem um risco adicional no futuro de pacientes tratados com $\mathrm{GH}$. A relação do uso de $\mathrm{GH}$ com a presença de tumores foi estabelecida em estudos in vitro que observaram que a utilização de IGF-1 é capaz de aumentar a proliferação de células tumorais em cultura. Não há evidências que assegurem que o $\mathrm{GH}$ determine a mudança de diferenciação 
de células normais para células neoplásicas. Achados indiretos foram apontados em pacientes acromegálicos, os quais apresentam maior prevalência de tumores intestinais. Deve-se ressaltar que estes pacientes estão submetidos a longos períodos de concentrações muito aumentadas de GH e IGF-1, habitualmente diferentes das determinadas com o uso de doses terapêuticas adequadas. Existe a possibilidade de associação com hipertrofia das amígdalas, a qual deve ser inspecionada rotineiramente durante o exame físico. A associação do GH com o GnRHa exige a observação evolutiva da massa óssea. A aceleração do crescimento pelo uso de GH pode evidenciar ou agravar uma escoliose subjacente.

\section{COMENTÁRIOS FINAIS}

O diagnóstico da BEI é sempre de exclusão e a reinvestigação etiológica deve ser praticada ao longo do acompanhamento clínico.

$\mathrm{O}$ uso de GH em pacientes com BEI é seguro e efetivo, porém tem eficácia relacionada a critérios clínicos e laboratoriais, que devem ser considerados na decisão da indicação terapêutica.

O paciente e os pais devem estar cientes de que o principal objetivo do tratamento é a obtenção da estatura final dentro da normalidade populacional e que traga conforto ao paciente na vida adulta. Deve-se evitar a expectativa de grande ganho estatural com o uso de GH em pacientes com BEI, visto que este resultado é pouco freqüente em pacientes habitualmente tratados com esta indicação.

Os aspectos éticos do uso de GH em pacientes com BEI incluem mostrar aos familiares a relação custo/benefício do tratamento com GH (21). Em nosso meio, os familiares de pacientes com BEI arcam com todas as despesas do tratamento e o preço final do GH pode ainda ser considerado muito elevado. Supondo-se que o peso médio do paciente a ser tratado seja de $30 \mathrm{~kg}$ e que o custo de 3 UI ( $1 \mathrm{mg}$ ) seja ao redor de $\mathrm{R} \$ 60$, podemos calcular que o uso da dose média de $0,15 \mathrm{UI} / \mathrm{kg} /$ dia $(0,05 \mathrm{mg} / \mathrm{kg} /$ dia) determine o gasto anual de cerca de R $\$ 33$ mil. Destacar que para que o tratamento tenha efeito significante sobre a estatura final deve ser oferecido $\mathrm{GH}$ por vários anos, em especial até depois do estirão puberal. Este é, sem dúvida, um aspecto que inviabiliza o tratamento para a maior parte de nossa população e seleciona de modo pouco ético, por um critério econômico, os pacientes com BEI que poderão ser beneficiados.

\section{REFERÊNCIAS}

1. Kochi C, Longui CA. Crescimento deficiente e uso terapêutico do hormônio de crescimento. In: Monte O, Longui CA, Calliari LEP, Kochi C. editors. Endocrinologia para o pediatra. $3^{\text {a }}$ ed. Rio de Janeiro: Atheneu; 2006.

2. Kemp SF, Kuntze J, Attie KM, Maneatis T, Butler S, Frane J, et al. Efficacy and safety results of long-term growth hormone treatment of idiopathic short stature. J Clin Endocrinol Metab. 2005;90:5247-53.

3. Cabezudo JID, Lezcano AC, Núñez CJV, Longas AF, Boutheliere RG, Arias MP. Talla baja idiopática: definición y tratamiento. An Pediatr (Barc). 2006;64(4):360-4.

4. Wit JM, Clayton PE, Rogol AD, Savage MO, Saenger PH, Cohen P. Idiopathic short stature: definition, epidemiology, and diagnostic evaluation. Growth Horm IGF Res. 2008;18(2):89-110.

5. Martinelli CE, Sader Milani S, Previato JK, Figueira M, Rangel Montenegro AP, Miraki-Moud F, et al. Final height in patients with idiopathic short stature and high growth hormone responses to stimulation tests. Horm Res. 2007;67(5):224-30.

6. Sjoberg M, Salazar T, Espinosa C, Dagnino A, Avila A, Eggers $M$, et al. Study of GH sensitivity in chilean patients with idiopathic short stature. J Clin Endocrinol Metab. 2001;86: 4375-81.

7. Rosenfeld RG. Pharmacogenomics and pharmacoproteomics in the evaluation and management of short stature. Eur J Endocrinol. 2007;157:S27-31.

8. Longui CA. Previsão da estatura final - acertando no "alvo". Arq Bras Endocrinol Metab. 2003;47(6):636-7.

9. Bryant J, Baxter L, Cave CB, Milne R. Recombinant growth hormone for idiopathic short stature in children and adolescents. Cochrane Database Syst Rev. 2007;18(3).

10. Quigley CA. Growth hormone treatment of non-growth hormone-deficient growth disorders. Endocrinol Metab Clin North Am. 2007;36(1):131-86.

11. Bakker B, Frane J, Anhalt H, Lippe B, Rosenfeld RG. Height velocity targets from the national cooperative growth study for first-year growth hormone responses in short children. $J$ Clin Endocrinol Metab. 2008;93(2):352-7.

12. Loche S, Casini MR, Ubertini GM, Cappa M. Growth hormone treatment in non-growth hormone-deficient short children. J Endocrinol Invest. 2005;28:193-8.

13. Leschek EW, Rose SR, Yanovski JA, Troendle JF, Quigley CA, Chipman JJ, et al. Effect of growth hormone treatment on adult height in peripubertal children with idiopathic short stature: a randomized, double-blind, placebo-controlled trial. $J$ Clin Endocrinol Metab. 2004;89:3140-8.

14. Crowe JA, Rekers-Mombarg LTM, Robling K, Wolka AM, Cutler Jr. GB, Wit JM. Effect of growth hormone dose on bone maturation and puberty in children with idiopathic short stature. $J$ Clin Endocrinol Metab. 2006;91:169-75.

15. Wit JM, Reiter EO, Ross JL, Saenger PH, Savage MO, Rogol $A D$, et al. Idiopathic short stature: management and growth hormone treatment. Growth Horm IGF Res. 2008;18(2):111-35. 
16. Ranke MB, Lindberg A, Price DA, Darendeliler F, AlbertssonWikland $K$, Wilton $P$, et al. Age at growth hormone therapy start and first-year responsiveness to growth hormone are major determinants of height outcome in idiopathic short stature. Horm Res. 2007;68(2):53-62.

17. Toumba M, IBacopoulou I, Savva SC, Skordis N. Efficacy of combined treatment with growth hormone and gonadotropin releasing hormone analogue in children with poor prognosis of adult height. Indian J Ped. 2007;44:497-502.

18. Damiani D, Damiani, D. Pharmacological management of children with short stature: the role of aromatase inhibitors. J Pediatr. 2007;83(5):S172-7.

19. Hero M, Ankarberg-Lindgren C, Taskinen M, Dunkel L. Blockade of oestrogen biosynthesis in peripubertal boys: effects on lipid metabolism, insulin sensitivity, and body composition. Eur J Endocrinol. 2006;155:453-60.
20. Damaceno FL, Santili C, Longui CA. Normal reference values of Southwick's anteroposterior angle in prepubertal and pubertal normal adolescents. J Pediatr Orthop B. 2007;16(6):389-92.

21. Lee JM, Davis MM, Clark SJ, Hofer TP, Kemper AR. Estimated cost-effectiveness of growth hormone therapy for idiopathic short stature. Arch Pediatr Adolesc Med. 2006;160:263-9.

Endereço para correspondência:

Carlos Alberto Longui

Departamento de Ciências Fisiológicas da FCMSC

Rua Cesário Mota Junior 61, -Vila Buarque

01223-020, Sao Paulo, SP

E-mail: carloslongui@msn.com 